\title{
REPRESENTATIONS OF UNIPOTENT GROUPS OVER LOCAL FIELDS AND GUTKIN'S CONJECTURE
}

\author{
Mitya BoyARCHENKO
}

\begin{abstract}
Let $F$ be a finite field or a local field of any characteristic. If $A$ is a finite dimensional associative nilpotent algebra over $F$, the set $1+A$ of all formal expressions of the form $1+x$, where $x \in A$, is a locally compact group with the topology induced by the standard one on $F$ and the multiplication $(1+x) \cdot(1+y)=1+(x+y+x y)$. We prove a result conjectured by E. Gutkin in 1973: every unitary irreducible representation of $1+A$ can be obtained by unitary induction from a 1-dimensional unitary character of a subgroup of the form $1+B$, where $B \subset A$ is an $F$-subalgebra. In the case where $F$ is local and nonarchimedean we also establish an analogous result for smooth irreducible representations of $1+A$ over $\mathbb{C}$ and show that every such representation is admissible and carries an invariant Hermitian inner product.
\end{abstract}

\section{Introduction}

Let $F$ be a self-dual field, that is, a finite field, or $\mathbb{R}$, or $\mathbb{C}$, or a finite extension of $\mathbb{Q}_{p}$, or a field $\mathbb{F}_{q}((t))$ of formal Laurent series in one variable over a finite field. We equip $F$ with the natural topology, making it a locally compact topological field (for a finite field, the topology is discrete). The term "self-dual" is explained by the following observation. Fix a nontrivial unitary character $\psi:(F,+) \longrightarrow \mathbb{C}^{\times}$of the additive group of $F$. For each $a \in F$, define $\psi_{a}(x)=\psi(a x)$ for all $x \in F$. Then the map $a \mapsto \psi_{a}$ is a topological isomorphism between $(F,+)$ and its Pontryagin dual.

Let $A$ be an associative $F$-algebra, which is not assumed to be unital. For each $m \geq 1$, write $A^{m}$ for the subspace of $A$ spanned by all elements of the form $a_{1} a_{2} \cdots a_{m}$, where $a_{j} \in A$. We say that $A$ is nilpotent if $A^{m}=0$ for some $m \geq 1$. In this case the set $1+A$ of all formal expressions of the form $1+x$, where $x \in A$, is a group under the operation $(1+x)(1+y)=1+(x+y+x y)$. (For instance, if $A^{2}=0$, then $1+A$ is identified with the additive group of $A$.)

Now suppose that $A$ is a finite dimensional associative nilpotent algebra over $F$. Then $A$ inherits a natural topology from $F$, and $1+A$ becomes a locally compact (Hausdorff) and second countable topological group. Moreover, it is unimodular. If $B$ is an $F$-subalgebra of $A$, then $1+B$ can be viewed as a closed subgroup of $1+A$.

In this situation, E. Gutkin formulated in [6] the following statement:

Theorem 1.1. Let $\pi: 1+A \longrightarrow U(\mathcal{H})$ be a unitary irreducible representation. Then there exist an $F$-subalgebra $B \subset A$ and a unitary character $\alpha: 1+B \longrightarrow \mathbb{C}^{\times}$such that $\pi \cong u-\operatorname{Ind}_{1+B}^{1+A} \alpha$.

Received by the editors June 30, 2010. 
In this statement, $U(\mathcal{H})$ denotes the group of unitary operators on a complex Hilbert space $\mathcal{H}$, and $\mathrm{u}$-Ind denotes the operation of unitary induction (see $\S 4.3 .3$ ).

The original motivation behind Theorem 1.1 was the following application:

Corollary 1.2. If $F=\mathbb{F}_{q}$ is a finite field with $q$ elements and $A$ is a finite dimensional associative nilpotent algebra over $\mathbb{F}_{q}$, the dimension of every complex irreducible representation of the finite group $1+A$ is a power of $q$.

This result follows from Theorem 1.1, because, in the situation of Corollary 1.2, if $B \subset A$ is a subalgebra, then the index of $1+B$ in $1+A$ equals $q^{\operatorname{dim}(A)-\operatorname{dim}(B)}$, which is a power of $q$.

As an example, fix an integer $n \geq 2$ and let $A$ be the algebra of strictly uppertriangular matrices of size $n$ over $\mathbb{F}_{q}$ (under the usual operations of matrix addition and matrix multiplication). Then $1+A$ can be identified with the group $U L_{n}\left(\mathbb{F}_{q}\right)$ of unipotent upper-triangular matrices of size $n$ over $\mathbb{F}_{q}$. In 1960 G. Higman asked whether the dimension of every (complex) irreducible representation of $U L_{n}\left(\mathbb{F}_{q}\right)$ is a power of $q$ (thus Corollary 1.2 yields an affirmative answer). Later this question was advertised and popularized by J. Thompson and A.A. Kirillov, among others, and eventually it led Gutkin to introduce the more general groups of the form $1+A$ and formulate the more precise Theorem 1.1 in [6].

To the best of our knowledge, the first complete proof of Corollary 1.2 was given by I.M. Isaacs [8] in 1995, whereas the first complete proof of Theorem 1.1 when $F$ is a finite field was given by Z. Halasi [7] in 2004. Unfortunately, Halasi's proof relies on the result of [8]; in particular, it uses a counting argument, and it is not clear how to adapt this argument to the case where $F$ is a local field.

When $F$ is a local field of characteristic 0 (that is, $\mathbb{R}$ or $\mathbb{C}$ or a finite extension of $\mathbb{Q}_{p}$ ), one can prove Theorem 1.1 using the orbit method for unipotent groups over $\mathbb{R}$ $[9]$ or over $\mathbb{Q}_{p}[10]$. We briefly explain the idea of this approach in $\S 2.2$.

The first goal of this article is to give a proof of Theorem 1.1 for an arbitrary self-dual field $F$, in a way that is independent of the characteristic of $F$ (see $\S 5.5$ and Remark 5.5). An important ingredient in the proof is a certain result on commutators in groups of the form $1+A$ that was first proved in [7]. We review it in $\S 3.2$.

One can also formulate Gutkin's conjecture in a different setting. Namely, suppose that $F$ is a nonarchimedean local field. Then the topological group $1+A$ is totally disconnected: there exists a basis of neighborhoods of the identity element of $1+A$ consisting of compact open subgroups. Thus it is natural to study smooth complex representations of $1+A$. Our second goal is to prove the following result:

Theorem 1.3. Let $F$ be a nonarchimedean local field, and let $\pi: 1+A \rightarrow G L(V)$ be a smooth irreducible representation. Then $\pi$ is admissible and unitarizable. Moreover, there exist an $F$-subalgebra $B \subset A$ and a smooth homomorphism $\alpha: 1+B \longrightarrow \mathbb{C}^{\times}$such that $\pi \cong \operatorname{Ind}_{1+B}^{1+A} \alpha$ and the natural map $c-\operatorname{Ind}_{1+B}^{1+A} \alpha \longrightarrow \operatorname{Ind}_{1+B}^{1+A} \alpha$ is an isomorphism.

In this statement, $G L(V)$ denotes the group of all linear automorphisms of a vector space $V$ over $\mathbb{C}$, and Ind and $\mathrm{c}$-Ind denote the operations of smooth induction and smooth induction with compact supports, respectively. All the terminology and 
notation used in Theorem 1.3 is reviewed in Section 4. The theorem itself is proved in $\S 5.4$.

It is natural to ask whether, if $\mathbb{G}$ is any unipotent algebraic group over a nonarchimedean local field $F$, then every smooth complex irreducible representation of $\mathbb{G}(F)$ is admissible and unitarizable. At present an affirmative answer is known when char $F=0$ thanks to $[11,12]$. The case char $F>0$ is open.

All the main ideas of our approach to Theorems 1.1 and 1.3 can already be explained in the case where $F$ is a finite field; at the same time, in this setting one avoids various technical complications that arise in the study of infinite dimensional (smooth or unitary) representations. Thus, for clarity, we treat this case first in $\S 2.3$.

Section 3 is essentially independent of, and different in spirit from, the rest of the article. In it we apply the techniques developed by Halasi in [7] to prove Corollary 2.3 , which is the key algebraic input in our proofs of Theorems 1.1 and 1.3.

In Section 4 we review the basics of $p$-adic representation theory and conclude by stating two theorems due to F. Rodier [11] that are crucial for our arguments. Finally, the complete proofs of Theorems 1.1 and 1.3 are presented in Section 5.

\section{Some special cases of Gutkin's conjecture}

We begin this section by introducing some general notation. Then we proceed to explain how Theorem 1.1 can be proved, in the case where $F$ is a local field of characteristic 0 , using the classical orbit method due to Kirillov in the case where $F$ is archimedean and to C.C. Moore in the case where $F$ is nonarchimedean. Finally we sketch a proof of Theorem 1.1 in the case where $F$ is finite. The proof is different from the ones that can be found in the literature [7], and contains all of the new ideas that allow us to prove Theorems 1.1 and 1.3 in full generality.

2.1. Notation. Throughout this paper, $(\cdot, \cdot)$ denotes the group commutator and $[\cdot, \cdot]$ denotes the Lie algebra commutator. We enunciate this in $\S \S 2.1 .1-2.1 .2$.

2.1.1. Let $\Gamma$ be a group. If $S_{1}, S_{2} \subset \Gamma$ are subsets, then $\left(S_{1}, S_{2}\right)$ denotes the subgroup of $\Gamma$ generated by all elements of the form $g h g^{-1} h^{-1}$ with $g \in S_{1}$ and $h \in S_{2}$.

2.1.2. Let $A$ be an associative (not necessarily unital) ring. If $x, y \in A$, then $[x, y] \stackrel{\text { def }}{=} x y-y x$. If $S_{1}, S_{2} \subset A$ are subsets, then $\left[S_{1}, S_{2}\right]$ denotes the additive subgroup of $A$ generated by all elements of the form $[x, y]$ with $x \in S_{1}$ and $y \in S_{2}$.

2.1.3. If $A$ is an associative ring, the two-sided ideals $A^{m} \subset A$ for all $m \geq 1$ and the notion of what it means for $A$ to be nilpotent are defined as in the Introduction.

2.1.4. Let $A$ be a nilpotent associative ring. The corresponding group $1+A$ is defined as in the Introduction. If $I \subset A$ is a subring, then $1+I$ is naturally identified with a subgroup of $1+A$. If $I$ is a two-sided ideal of $A$, then $1+I$ is a normal subgroup of $1+A$ (the converse is usually false), and the quotient group $(1+A) /(1+I)$ can be naturally identified with $1+(A / I)$, where $A / I$ is the corresponding quotient ring. All these remarks are used implicitly in what follows. 
2.2. Local fields of characteristic 0 . Let us first settle Gutkin's original conjecture (Theorem 1.1) in the case where the base field is $\mathbb{R}$ or $\mathbb{C}$. We will use the classical orbit method due to Kirillov [9] together with an observation that we originally learned from a work of C.A.M. André [2].

Let $F$ be a local archimedean field, and let $A$ be a finite dimensional associative nilpotent algebra over $F$. There is no loss of generality in assuming that $F=\mathbb{R}$ (if $F=\mathbb{C}$ we can view $A$ as an algebra of dimension $2 \cdot \operatorname{dim}_{\mathbb{C}} A$ over $\mathbb{R}$ ). We can view $1+A$ as a nilpotent Lie group, and its Lie algebra can be naturally identified with $A$ (where the latter is equipped with the commutator bracket $[a, b]=a b-b a$ ) by means of the exponential map:

$$
\exp : A \stackrel{\sim}{\longrightarrow} 1+A, \quad x \longmapsto 1+x+\frac{x^{2}}{2 !}+\frac{x^{3}}{3 !}+\cdots
$$

(here we use both the fact that $\mathbb{R}$ has characteristic 0 and the assumption that $A$ is nilpotent).

If $G$ is a connected and simply connected nilpotent Lie group, $\mathfrak{g}$ is its Lie algebra, and $f: \mathfrak{g} \longrightarrow \mathbb{R}$ is a linear map, a polarization of $f$ is a Lie subalgebra $\mathfrak{h} \subset \mathfrak{g}$ that satisfies $f([\mathfrak{h}, \mathfrak{h}])=0$ and is maximal among all possible subspaces of $\mathfrak{g}$ satisfying this property. Kirillov proved in [9] that every linear $f: \mathfrak{g} \longrightarrow \mathbb{R}$ has a polarization $\mathfrak{h}$, and the corresponding induced representation $\mathrm{u}-\operatorname{Ind}_{H}^{G} \chi_{f}$ of $G$ is irreducible. Here $H \subset G$ is the subgroup corresponding to $\mathfrak{h}$ and $\chi_{f}=\exp (i f)$ is the unitary character of $H$ defined by $f$. Moreover, Kirillov showed that every unitary irreducible representation of $G$ is unitarily equivalent to $\mathrm{u}-\operatorname{Ind}_{H}^{G} \chi_{f}$ for suitable $f$ and $\mathfrak{h}$.

In view of these results, we see that Theorem 1.1 for $F=\mathbb{R}$ follows from

Lemma 2.1. Let $A$ be a finite dimensional associative nilpotent algebra over a field $\mathfrak{k}$, and let $f: A \longrightarrow \mathfrak{k}$ be a linear functional. Then there exists an (associative) $\mathfrak{k}$ subalgebra $B \subset A$ such that, as a Lie subalgebra of $A$ with respect to the commutator bracket, $B$ is a polarization of $f$.

For the proof, see [2] or [4, Theorem D.13].

Next let us consider Theorem 1.1 in the case where $F$ is a finite extension of $\mathbb{Q}_{p}$. As before, we may assume that $F=\mathbb{Q}_{p}$, and then we only need to repeat the proof of the case $F=\mathbb{R}$ almost verbatim, replacing the reference to [9] with a reference to [10], where the orbit method for nilpotent Lie groups over $\mathbb{Q}_{p}$ was developed.

Remark 2.2. We were somewhat sketchy in this treatment of Theorem 1.1 for local fields of characteristic 0 because the argument we give in $\S 5.5$ below is also valid in this setting, and provides a different approach that does not directly rely on the results of $[9,10]$. Of course, some of the ideas we use are still directly inspired by the works of G. Mackey, as well as Kirillov, Moore and the other pioneers of the orbit method for nilpotent groups.

2.3. Finite fields. Let us conclude the section by sketching a proof of Theorem 1.1 in the case where $F$ is a finite field. We will be brief, because Section 5 below presents a self-contained proof of Theorems 1.1 and 1.3 that is valid both for finite fields and for local nonarchimedean fields. On the other hand, the argument we sketch here already contains all of the important ideas behind the proofs of Theorems 1.1 and 
1.3 in full generality. Therefore we would like to present these ideas in an elementary context before reviewing the technical machinery of Section 4.

The proof is based on the following crucial result.

Corollary 2.3. Let $\mathfrak{k}$ be an arbitrary field, and let $A$ be an associative nilpotent algebra over $\mathfrak{k}$. Fix an integer $m \geq 2$, let $\zeta: 1+A^{m} \longrightarrow \mathbb{C}^{\times}$be a homomorphism that is invariant under the conjugation action of $1+A$ on $1+A^{m}$, and define

$$
C_{\zeta}:(1+A) \times\left(1+A^{m-1}\right) \longrightarrow \mathbb{C}^{\times}
$$

by $C_{\zeta}(g, h)=\zeta\left(g h g^{-1} h^{-1}\right)$. Then

(a) $C_{\zeta}$ factors through a map

$$
\bar{C}_{\zeta}:\left(1+\left(A / A^{2}\right)\right) \times\left(1+\left(A^{m-1} / A^{m}\right)\right) \longrightarrow \mathbb{C}^{\times} ;
$$

and

(b) the map $\bar{C}_{\zeta}$ is $\mathfrak{k}$-bilinear in the following sense:

- $\bar{C}_{\zeta}\left(1+x_{1}+x_{2}, 1+y\right)=\bar{C}_{\zeta}\left(1+x_{1}, 1+y\right) \cdot \bar{C}_{\zeta}\left(1+x_{2}, 1+y\right)$ for all $x_{1}, x_{2} \in A / A^{2}$ and all $y \in A^{m-1} / A^{m}$;

- $\bar{C}_{\zeta}\left(1+x, 1+y_{1}+y_{2}\right)=\bar{C}_{\zeta}\left(1+x, 1+y_{1}\right) \cdot \bar{C}_{\zeta}\left(1+x, 1+y_{2}\right)$ for all $x \in A / A^{2}$ and all $y_{1}, y_{2} \in A^{m-1} / A^{m}$;

- $\bar{C}_{\zeta}(1+\lambda x, 1+y)=\bar{C}_{\zeta}(1+x, 1+\lambda y)$ for all $\lambda \in \mathfrak{k}, x \in A / A^{2}, y \in A^{m-1} / A^{m}$.

This corollary follows immediately from Proposition 3.1 below $^{1}$, which is proved in Section 3 (the proof we give there is independent of any of the other results of this article).

Now let $F$ be a finite field, let $A$ be a finite dimensional associative nilpotent $F$-algebra, and let $\pi: 1+A \longrightarrow G L(V)$ be an irreducible representation of the finite group $1+A$, where $V$ is a finite dimensional vector space over $\mathbb{C}$. Since $A$ is nilpotent, there exists a smallest integer $m \geq 1$ with the property that $\pi\left(1+A^{m}\right)$ consists of scalar operators. We may assume that $m \geq 2$, since otherwise $\pi$ is already 1-dimensional and there is nothing to prove.

We obtain a homomorphism $\zeta: 1+A^{m} \longrightarrow \mathbb{C}^{\times}$such that $\pi(g)=\zeta(g) \cdot \mathrm{id}_{V}$ for all $g \in 1+A^{m}$. The construction of $\zeta$ implies that it is invariant under the conjugation action of $1+A$. Hence Corollary 2.3 can be applied. Let $\bar{C}_{\zeta}$ be defined as in that corollary and note that $\bar{C}_{\zeta}$ is not identically 1 , since otherwise the minimality of $m$ would be contradicted.

Since $1+\left(A / A^{2}\right)$ and $1+\left(A^{m-1} / A^{m}\right)$ are naturally identified with the additive groups of $A / A^{2}$ and $A^{m-1} / A^{m}$, respectively, we can view $\bar{C}_{\zeta}$ as a homomorphism of abelian groups

$$
\Phi_{\zeta}: A / A^{2} \longrightarrow\left(A^{m-1} / A^{m}\right)^{*} \stackrel{\text { def }}{=} \operatorname{Hom}\left(A^{m-1} / A^{m}, \mathbb{C}^{\times}\right) .
$$

Since $F$ is finite and $\operatorname{dim}_{F}\left(A^{m-1} / A^{m}\right)<\infty$, we can further identify $\left(A^{m-1} / A^{m}\right)^{*}$ with the additive group of the $F$-vector space dual of $A^{m-1} / A^{m}$. With these identifications, the content of Corollary 2.3(b) is that $\Phi_{\zeta}$ is a linear map of F-vector spaces.

\footnotetext{
${ }^{1}$ Observe that $\zeta$ is invariant under $(1+A)$-conjugation if and only if $\zeta$ is trivial on the commutator $\left(1+A, 1+A^{m}\right)$
} 
Since $\Phi_{\zeta}$ is not identically zero by construction, we can find a 1-dimensional $F$ vector subspace $L \subset A^{m-1} / A^{m}$ such that the composition

$$
A / A^{2} \stackrel{\Phi_{\zeta}}{\longrightarrow}\left(A^{m-1} / A^{m}\right)^{*} \longrightarrow L^{*}
$$

is surjective, where the second map is given by restriction. Let $A_{1} \subset A$ denote the preimage of the kernel of the above composition; then $A_{1}$ is a codimension 1 two-sided ideal of $A$. Also, let $U \subset A^{m-1}$ denote the preimage of $L$; then $U$ is a two-sided ideal of $A$. It is easy to see that $\left.\zeta\right|_{(1+U, 1+U)} \equiv 1$; or, equivalently, that $U \subset A_{1}$. (See Lemma 5.1 for the proof.)

Write $G=1+A, H=1+A_{1}, N=1+U$. Then $N \subset H \subset G$, and both $N$ and $H$ are normal subgroups of $G$. Let $\rho$ be an irreducible summand of the restriction $\left.\pi\right|_{H}$. If we show that $\operatorname{Ind}_{H}^{G} \rho$ is irreducible, Frobenius reciprocity will imply that $\pi \cong \operatorname{Ind}_{H}^{G} \rho$ and the proof will be complete ${ }^{2}$.

To check that $\operatorname{Ind}_{H}^{G} \rho$ is irreducible, we apply Mackey's irreducibility criterion. Suppose $g \in G$ is such that $g$ leaves the isomorphism class of $\rho$ invariant. Since $\zeta$ annihilates the commutator $(H, N)$ by construction, Schur's lemma implies that $\rho$ acts on $N$ via some scalar $\chi: N \longrightarrow \mathbb{C}^{\times}$. We then necessarily have $\left.\chi\right|_{1+A^{m}}=\zeta$, and the definition of $H$ implies that $H$ is precisely the stabilizer of $\chi$ under the conjugation action of $G$ on $N$. Since we assumed that $g$ leaves the isomorphism class of $\rho$ invariant, a fortiori, $g$ must leave $\chi$ invariant, whence $g \in H$. This verifies the hypothesis of Mackey's irreducibility criterion (in the special case where one is inducing from a normal subgroup of a finite group), and the proof is complete.

Remark 2.4. The proof sketched above has some ideas in common with the proof of Theorem 1.1 that was presented in [7] in the case where $F$ is a finite field. However, that proof also contains some ingredients that seem to have no analogues in the case where $F=\mathbb{F}_{q}((t))$. We were able to circumvent this difficulty by using Corollary 2.3.

\section{Commutators in groups of the form $1+A$}

This section is essentially independent of the rest of the article, in the sense that only the statement of Proposition 3.1 below is used elsewhere in the text, and the proofs we present here rely exclusively on the results and techniques developed in [7].

3.1. The commutator pairing. The main goal of this section is the following

Proposition 3.1. Let $\mathfrak{k}$ be an arbitrary field, and let $A$ be an associative nilpotent algebra over $\mathfrak{k}$. Fix an integer $m \geq 2$, put

$$
Q=\left(1+A^{m}\right) /\left(1+A, 1+A^{m}\right)
$$

and define

$$
C:(1+A) \times\left(1+A^{m-1}\right) \longrightarrow Q
$$

by letting $C(g, h)$ be the image of $g h g^{-1} h^{-1} \in 1+A^{m}$ in $Q$. Then

\footnotetext{
${ }^{2}$ By induction on $\operatorname{dim}_{F} A$, we may assume that Gutkin's conjecture holds for $A_{1}$ in place of $A$.
} 
(a) C factors through a map

$$
\bar{C}:\left(1+\left(A / A^{2}\right)\right) \times\left(1+\left(A^{m-1} / A^{m}\right)\right) \longrightarrow Q ;
$$

and

(b) the map $\bar{C}$ is $\mathfrak{k}$-bilinear in the following sense:

- $\bar{C}\left(1+x_{1}+x_{2}, 1+y\right)=\bar{C}\left(1+x_{1}, 1+y\right) \cdot \bar{C}\left(1+x_{2}, 1+y\right)$ for all $x_{1}, x_{2} \in A / A^{2}$ and all $y \in A^{m-1} / A^{m}$;

- $\bar{C}\left(1+x, 1+y_{1}+y_{2}\right)=\bar{C}\left(1+x, 1+y_{1}\right) \cdot \bar{C}\left(1+x, 1+y_{2}\right)$ for all $x \in A / A^{2}$ and all $y_{1}, y_{2} \in A^{m-1} / A^{m}$;

- $\bar{C}(1+\lambda x, 1+y)=\bar{C}(1+x, 1+\lambda y)$ for all $\lambda \in \mathfrak{k}, x \in A / A^{2}, y \in A^{m-1} / A^{m}$.

This result will be proved in $\S 3.3$ below after reviewing some preliminaries.

3.2. Free nilpotent algebras. Let $R$ be an arbitrary associative, commutative, unital ring, let $X$ be a set, and let $n \in \mathbb{N}$. If $A$ is any nilpotent associative algebra over $R$, we call the nilpotence class of $A$ the smallest integer $k$ such that $A^{k}=0$. The free associative nilpotent algebra of nilpotence class $n$ generated by the set $X$ over $R$ is denoted by $F_{R}(n, X)$ and is constructed as follows. First let $\operatorname{span}_{R}(X)$ denote the free $R$-module generated by the set $X$, and let

$$
T=R\langle X\rangle=\bigoplus_{m \geq 0}\left(\operatorname{span}_{R}(X)\right)^{\otimes m}
$$

be the free associative unital $R$-algebra on the set $X$. It is naturally graded, and we will write

$$
T_{j}=\left(\operatorname{span}_{R}(X)\right)^{\otimes j} \quad \text { and } \quad T_{\geq m}=\bigoplus_{j \geq m} T_{j} .
$$

Writing $J=F_{R}(n, X)$ for simplicity, we define

$$
J=T_{\geq 1} / T_{\geq n}
$$

Thus $J$ inherits a grading from $T$. It is clear that $J$ satisfies a universal property with respect to all set maps from $X$ to associative nilpotent algebras over $R$ of nilpotence class $\leq n$.

Proposition 3.2. Suppose that $R$ is an integral domain of characteristic 0 . If $J=$ $F_{R}(n, X)$ for some set $X$ and some integer $n \geq 1$, then for all $k \geq 2$, we have

$$
(1+J, 1+J) \cap\left(1+J^{k}\right)=\left(1+J, 1+J^{k-1}\right) .
$$

Proof. When $R=\mathbb{Z}$ this is [7, Lem. 2.5]. In general one can check, line-by-line, that all the arguments in $\S 2$ of op. cit. remain valid if one replaces $\mathbb{Z}$ by an arbitrary integral domain $R$ of characteristic 0 and $\mathbb{Q}$ by the field of fractions of $R$.

Theorem 3.3. If $A$ is an arbitrary associative nilpotent ring, then for all $m, n \in \mathbb{N}$, we have

$$
\left(1+A^{m}, 1+A^{n}\right) \subseteq\left(1+A, 1+A^{m+n-1}\right) .
$$

This is precisely Theorem 1.4 in op. cit. 
3.3. Proof of Proposition 3.1. We begin with

Lemma 3.4. Let $N$ be an associative ring, and let $m \geq 2$ be an integer such that $N^{m+1}=0$. If $x \in N$ and $y \in N^{m-1}$, then $(1+x)(1+y)(1+x)^{-1}(1+y)^{-1}=1+[x, y]$ in $1+N$.

Proof. We have $x y x=0=y x^{2}$ in $N$. Using this observation, we calculate

$$
\begin{aligned}
(1+x)(1+y)(1+x)^{-1} & =1+(1+x) y(1+x)^{-1} \\
& =1+(1+x) y(1-x)=1+y+x y-y x .
\end{aligned}
$$

Since $m \geq 2$, we also have $x y^{2}=y x y=0$. Thus

$$
(1+y+x y-y x) \cdot(1+y)^{-1}=1+(x y-y x)(1+y)^{-1}=1+x y-y x,
$$

as claimed.

Now Proposition 3.1(a) follows from the fact that $\left(1+A^{2}, 1+A^{m-1}\right) \subset(1+A, 1+$ $\left.A^{m}\right)$, which is a special case of Theorem 3.3.

Let us prove the first assertion of Proposition 3.1(b). For the purposes of this argument, let us introduce the notation $\beta(g, h)=g h g^{-1} h^{-1}$, where $g, h$ are elements of some group. The first assertion of Proposition 3.1(b) amounts to the following claim: if $x_{1}, x_{2} \in A$ and $y \in A^{m-1}$, then

$$
\beta\left(1+x_{1}+x_{2}, 1+y\right) \cdot \beta\left(1+x_{1}, 1+y\right)^{-1} \cdot \beta\left(1+x_{2}, 1+y\right)^{-1} \in\left(1+A, 1+A^{m}\right)
$$

To see this, let us write $y=\sum_{j=1}^{N} a_{1, j} a_{2, j} \cdots a_{(m-1), j}$ for some elements $a_{i, j} \in A$, where $N$ is a positive integer, $1 \leq i \leq m-1$ and $1 \leq j \leq N$. (We can find such a presentation by the definition of $A^{m-1}$.) Introduce a set $X$ of formal symbols as follows:

$$
X=\left\{\widetilde{x}_{1}, \widetilde{x}_{2}\right\} \cup\left\{\widetilde{a}_{i, j}\right\}_{1 \leq i \leq m-1,1 \leq j \leq N} .
$$

Let $n$ be the nilpotence class of $A$ and consider $J=F r_{\mathbb{Z}}(n, X)$, the free associative nilpotent algebra of nilpotence class $n$ generated by the set $X$ over $\mathbb{Z}$ (see $\S 3.2$ ).

Let $\varphi: J \longrightarrow A$ be the homomorphism of associative rings that takes $\widetilde{x}_{i} \mapsto x_{i}$ $(i=1,2)$ and takes $\widetilde{a}_{i, j} \mapsto a_{i, j}$ for all $i, j$. Then we have $\varphi(\widetilde{y})=y$, where

$$
\widetilde{y}=\sum_{j=1}^{N} \widetilde{a}_{1, j} \widetilde{a}_{2, j} \cdots \widetilde{a}_{(m-1), j} \in J^{m-1} .
$$

Now consider the reduction of $J$ modulo $J^{m+1}$. Applying Lemma 3.4, we see that

$$
\begin{aligned}
\beta\left(1+\widetilde{x}_{1}+\widetilde{x}_{2}, 1+\widetilde{y}\right) & =1+\left[\widetilde{x}_{1}+\widetilde{x}_{2}, \widetilde{y}\right] \bmod J^{m+1}, \\
\beta\left(1+\widetilde{x}_{1}, 1+\widetilde{y}\right) & =1+\left[\widetilde{x}_{1}, \widetilde{y}\right] \bmod J^{m+1}
\end{aligned}
$$

and

$$
\beta\left(1+\widetilde{x}_{2}, 1+\widetilde{y}\right)=1+\left[\widetilde{x}_{2}, \widetilde{y}\right] \bmod J^{m+1}
$$


Since $\left[\widetilde{x}_{1}+\widetilde{x}_{2}, \widetilde{y}\right],\left[\widetilde{x}_{1}, \widetilde{y}\right],\left[\widetilde{x}_{2}, \widetilde{y}\right] \in J^{m}$, it follows that

$$
\beta\left(1+\widetilde{x}_{1}+\widetilde{x}_{2}, 1+\widetilde{y}\right)=\beta\left(1+\widetilde{x}_{1}, 1+\widetilde{y}\right) \cdot \beta\left(1+\widetilde{x}_{2}, 1+\widetilde{y}\right) \quad \bmod J^{m+1},
$$

or equivalently

$$
\beta\left(1+\widetilde{x}_{1}+\widetilde{x}_{2}, 1+\widetilde{y}\right) \cdot \beta\left(1+\widetilde{x}_{1}, 1+\widetilde{y}\right)^{-1} \cdot \beta\left(1+\widetilde{x}_{2}, 1+\widetilde{y}\right)^{-1} \in 1+J^{m+1} .
$$

Since the last expression belongs to $(1+J, 1+J)$ by definition, we see that it in fact belongs to $(1+J, 1+J) \cap\left(1+J^{m+1}\right)$. By virtue of Proposition 3.2, the last intersection is equal to $\left(1+J, 1+J^{m}\right)$; thus

$$
\beta\left(1+\widetilde{x}_{1}+\widetilde{x}_{2}, 1+\widetilde{y}\right) \cdot \beta\left(1+\widetilde{x}_{1}, 1+\widetilde{y}\right)^{-1} \cdot \beta\left(1+\widetilde{x}_{2}, 1+\widetilde{y}\right)^{-1} \in\left(1+J, 1+J^{m}\right) .
$$

Applying the homomorphism $\varphi: J \longrightarrow A$ to the last containment yields (3.1).

The proof of the second assertion of Proposition 3.1(b) is almost identical to the proof we just presented, so we will skip it.

Finally, the proof of the third assertion is similar, but contains an additional idea. Again, the assertion amounts to the following statement: if $x \in A, y \in A^{m-1}$ and $\lambda \in \mathfrak{k}$, then

$$
\beta(1+\lambda x, 1+y) \cdot \beta(1+x, 1+\lambda y)^{-1} \in\left(1+A, 1+A^{m}\right) .
$$

To prove this containment, we begin, as before, by writing

$$
y=\sum_{j=1}^{N} a_{1, j} a_{2, j} \cdots a_{(m-1), j}
$$

for suitable $a_{i, j} \in A$. Consider the set of formal symbols

$$
X=\{\widetilde{x}\} \cup\left\{\widetilde{a}_{i, j}\right\}_{1 \leq i \leq m-1,1 \leq j \leq N},
$$

and let $R=\mathbb{Z}[\widetilde{\lambda}]$ be the polynomial ring over $\mathbb{Z}$ in one variable $\tilde{\lambda}$. There is a unique ring homomorphism $R \longrightarrow \mathfrak{k}$ such that $\widetilde{\lambda} \mapsto \lambda$; by means of this homomorphism we can view $\mathfrak{k}$, and hence also $A$, as $R$-algebras.

Next let $J=F r_{R}(n, X)$ be the free associative nilpotent algebra of nilpotence class $n$ generated by the set $X$ over $R$ (where $n$ is the nilpotence class of $A$ ) and let $\varphi: J \longrightarrow A$ be the homomorphism of $R$-algebras determined by $\varphi(\widetilde{x})=x$ and $\varphi\left(\widetilde{a}_{i, j}\right)=a_{i, j}$ for all $i, j$.

From this point on the proof proceeds as before. Using Lemma 3.4 we deduce that

$$
\beta(1+\widetilde{\lambda} \widetilde{x}, 1+\widetilde{y})=\beta(1+\widetilde{x}, 1+\widetilde{\lambda} \widetilde{y}) \bmod J^{m+1},
$$

which implies that

$\beta(1+\widetilde{\lambda} \widetilde{x}, 1+\widetilde{y}) \cdot \beta(1+\widetilde{x}, 1+\widetilde{\lambda} \widetilde{y})^{-1} \in(1+J, 1+J) \cap\left(1+J^{m+1}\right)=\left(1+J, 1+J^{m}\right)$, where the last equality follows from Proposition 3.2. Applying $\varphi$ to the last containment yields (3.2), which completes the proof of Proposition 3.1. 


\section{Representations of totally disconnected groups}

This section is mostly a review of standard facts and definitions from the theory of smooth representations of locally compact totally disconnected topological groups. In $\S 4.7$ we reproduce some important results of F. Rodier [11], which play a crucial role in Section 5 below.

4.1. Representations of $\ell$-groups. In this article we will follow the terminology of [3]. In particular, we make the following

Definition 4.1. An $\ell$-group ${ }^{3}$ is a Hausdorff topological group $G$ such that the unit element $1 \in G$ has a neighborhood basis consisting of compact open subgroups of $G$.

A primary example of an $\ell$-group for us is the group $\mathbb{G}(F)$ of $F$-points of an algebraic group $\mathbb{G}$ over a local nonarchimedean field $F$. The topology on $\mathbb{G}(F)$ is induced by the usual topology on $F$. An $\ell$-group of the form $\mathbb{G}(F)$ is also second countable, which will be important for us.

It is convenient to isolate a special class of $\ell$-groups that plays an important role in [11] (on which our work relies heavily), but for which no term was introduced in op. cit.

Definition 4.2. An $\ell_{c}$-group is an $\ell$-group $G$ that is a filtered union of its compact open subgroups; in other words, every $g \in G$ is contained in a compact open subgroup of $G$, and any two such subgroups are together contained in a third such subgroup.

For instance, if $\mathbb{G}$ is a unipotent algebraic group over a local nonarchimedean field $F$, then $\mathbb{G}(F)$ is an $\ell_{c}$-group. In particular, groups of the form $1+A$, where $A$ is a finite dimensional associative nilpotent algebra over $F$, are $\ell_{c}$-groups.

For convenience, we recall that a smooth ${ }^{4}$ representation of an $\ell$-group $G$ is a pair $(\pi, V)$ consisting of a complex vector space $V$ and a homomorphism $\pi: G \longrightarrow G L(V)$ with the property that for each $v \in V$, the stabilizer $G_{v}=\{g \in G \mid \pi(g) v=v\}$ is open in $G$. By a standard abuse of notation we will sometimes denote a smooth representation $(\pi, V)$ by the single letter $\pi$ or $V$.

A morphism between smooth representations of $G$ is defined as a homomorphism of abstract representations, and the category of smooth representations of $G$ will be denoted by $\mathcal{R}(G)$.

A smooth representation $(\pi, V)$ of $G$ is said to be:

- irreducible if $V \neq 0$ and the only $\pi(G)$-invariant subspaces of $V$ are 0 and $V$;

- admissible if for every compact open subgroup $K \subset G$ the subspace

$$
V^{K}=\{v \in V \mid \pi(g) v=v \forall g \in K\}
$$

is finite dimensional;

- unitarizable if $V$ has a positive definite Hermitian inner product invariant under $\pi(G)$.

\footnotetext{
${ }^{3}$ Another common term is "l.c.t.d. group," which stands for "locally compact totally disconnected group."

"The adjective "algebraic" is used in [3] in place of "smooth."
} 
By an argument of Jacquet (cf. [3, 2.11]), Schur's lemma holds for second countable $\ell$-groups. More precisely, if $(\pi, V)$ is an irreducible smooth representation of a second countable $\ell$-group, then every linear operator $V \longrightarrow V$ that commutes with $\pi(G)$ is a scalar.

In Theorem 1.1 we need to deal with unitary representations (which are typically not smooth) of topological groups. Let us recall that a unitary representation of a topological group $G$ is a pair $(\pi, \mathcal{H})$ consisting of a Hilbert space $\mathcal{H}$ over $\mathbb{C}$ and a continuous homomorphism $\pi: G \longrightarrow U(\mathcal{H})$, where $U(\mathcal{H})$ is the group of unitary linear automorphisms of $\mathcal{H}$ equipped with the strong operator topology. In this case $(\pi, \mathcal{H})$ is said to be irreducible if $\mathcal{H} \neq 0$ and the only closed subspaces of $\mathcal{H}$ invariant under $\pi(G)$ are 0 and $\mathcal{H}$.

4.2. The smooth dual. If $(\pi, V)$ is an abstract representation ${ }^{5}$ of an $\ell$-group $G$, the subset $V^{\mathrm{sm}} \subset V$ consisting of vectors $v \in V$ for which the stabilizer $G_{v}$ is open is a $G$ subrepresentation of $V$, which by definition is smooth. In fact, the functor $V \longmapsto V^{\mathrm{sm}}$ from the category $\operatorname{Rep}(G)$ of abstract representations of $G$ to the category $\mathcal{R}(G)$ is right adjoint to the inclusion functor $\mathcal{R}(G) \hookrightarrow \operatorname{Rep}(G)$.

If $V$ is a smooth representation of $G$, form the contragredient representation $\operatorname{Hom}_{\mathbb{C}}(V, \mathbb{C})$ of $G$ as an abstract group. The smooth part $V^{\vee}:=\operatorname{Hom}_{\mathbb{C}}(V, \mathbb{C})^{\mathrm{sm}}$ is called the smooth dual of $V$. We also write $\pi^{\vee}: G \longrightarrow G L\left(V^{\vee}\right)$ for the corresponding homomorphism.

The relation between the notion of admissibility and smooth duality is explained by the following standard (and easy) statement.

Lemma 4.3. If $\pi$ is a smooth representation of an $\ell$-group $G$, then $\pi$ is admissible if and only if the canonical morphism $\pi \longrightarrow\left(\pi^{\vee}\right)^{\vee}$ is an isomorphism. In particular, if $\pi$ is a smooth irreducible admissible representation of $G$, then $\pi^{\vee}$ is also irreducible and admissible.

4.3. Induction functors. In this paper we use three induction functors for representations of $\ell$-groups. Let $G$ be an $\ell$-group, and let $H \subset G$ be a closed subgroup (in particular, $H$ is also an $\ell$-group).

4.3.1. Smooth induction. Suppose $(\rho, W)$ is a smooth representation of $H$. We can form the induced representation of $\rho$ from $G$ to $H$ in the sense of abstract groups. By definition, its underlying vector space $V_{\text {big }}$ consists of all functions $f: G \longrightarrow W$ that satisfy

$$
f(g h)=\rho(h)^{-1} \cdot f(g) \quad \forall g \in G, h \in H,
$$

and the $G$ action on $V_{\text {big }}$ is given by left translation:

$$
(g \cdot f)\left(g^{\prime}\right)=f\left(g^{-1} \cdot g^{\prime}\right) \quad \forall f \in V_{\text {big }}, g, g^{\prime} \in G .
$$

The representation $V_{\text {big }}^{\text {sm }}$ of $G$ will be denoted by $\operatorname{Ind}_{H}^{G} \rho$ or $\operatorname{Ind}_{H}^{G} W$. Thus we obtain a functor

$$
\operatorname{Ind}_{H}^{G}: \mathcal{R}(H) \longrightarrow \mathcal{R}(G),
$$

\footnotetext{
${ }^{5}$ That is, $\pi: G \longrightarrow G L(V)$ is a homomorphism of abstract groups.

${ }^{6}$ The notation $(\widetilde{\pi}, \widetilde{V})$ is used in [3] in place of $\left(\pi^{\vee}, V^{\vee}\right)$, but we prefer not to use the tilde symbol to avoid confusion with its other common uses.
} 
called smooth induction. Frobenius reciprocity follows formally: $\operatorname{Ind}_{H}^{G}$ is right adjoint to the restriction functor $\mathcal{R}(G) \longrightarrow \mathcal{R}(H)$.

4.3.2. Compact induction. In the setting of $\S 4.3 .1$, the subspace of $V_{\mathrm{big}}^{\mathrm{sm}}$ consisting of functions $f \in V_{\mathrm{big}}^{\mathrm{sm}}$ whose support is compact modulo $H$ affords a smooth $G$ subrepresentation of $\operatorname{Ind}_{H}^{G} \rho$, which we denote by c- $\operatorname{Ind}_{H}^{G} \rho$. Thus we obtain a functor $\mathrm{c}-\operatorname{Ind}_{H}^{G}: \mathcal{R}(H) \longrightarrow \mathcal{R}(G)$ together with a natural transformation $\mathrm{c}-\operatorname{Ind}_{H}^{G} \hookrightarrow \operatorname{Ind}_{H}^{G}$. One calls c-Ind ${ }_{H}^{G}$ the functor of induction with compact supports (or simply "compact induction").

We caution the reader that in [11], the notation $\operatorname{Ind}_{H}^{G}$ is used for the functor of induction with compact supports, while no notation for the smooth induction functor is introduced.

Remark 4.4. Suppose $G$ and $H$ are unimodular $\ell$-groups, which is the case in which we are mostly interested. (For example, if $\mathbb{G}$ and $\mathbb{H}$ are unipotent algebraic groups over a local field $F$, then $\mathbb{G}(F)$ and $\mathbb{H}(F)$ are unimodular $\ell$-groups.) Then there is a simple relationship between the functors $\mathrm{c}-\operatorname{Ind}_{H}^{G}$ and $\operatorname{Ind}_{H}^{G}$. Namely, if $\rho$ is any smooth representation of $H$, there is a natural isomorphism of $G$-representations between $\left(\mathrm{c}-\operatorname{Ind}_{H}^{G} \rho\right)^{\vee}$ and $\operatorname{Ind}_{H}^{G}\left(\rho^{\vee}\right)$.

A more general statement, where the unimodularity of $G$ or $H$ is not assumed, is proved in [3, Proposition 2.25(c)].

4.3.3. Unitary induction. Here we only consider the unimodular case, since it is simpler and it is the only one relevant for us. Let $G$ be a unimodular $\ell$-group, and let $H \subset G$ be a closed unimodular subgroup. Then the coset space $G / H$ has a $G$-invariant measure, which is unique up to scaling. Fix one such measure $\mu$.

Suppose $\rho: H \longrightarrow U(\mathcal{H})$ is a unitary representation of $H$, and let us denote the corresponding Hermitian inner product on $\mathcal{H}$ by $\langle\cdot, \cdot\rangle$. Given a measurable function $f: G \longrightarrow \mathcal{H}$ that satisfies $f(g h)=\rho(h) \cdot f(g)$ for all $g \in G$ and all $h \in H$, the function $\langle f, f\rangle: g \longmapsto\langle f(g), f(g)\rangle$ descends to the coset space $G / H$. One defines u- $\operatorname{Ind}_{H}^{G} \rho$ as the unitary representation of $G$ where:

- the underlying space consists of measurable functions $f: G \longrightarrow \mathcal{H}$ such that $f(g h)=\rho(h) \cdot f(g)$ for all $g \in G$ and all $h \in H$ and such that $\int_{G / H}\langle f, f\rangle d \mu<$ $\infty$;

- the inner product is defined by

$$
\left(f_{1}, f_{2}\right) \longmapsto \int_{G / H}\left\langle f_{1}, f_{2}\right\rangle d \mu
$$

- the $G$-action is given by left translation, as before.

4.4. Jacquet functors. If $G$ is an $\ell$-group and $(\pi, V)$ is a smooth representation of $G$, we denote by $J_{G}(\pi)$ the quotient of $V$ by the subspace spanned by all elements of the form $\pi(g) \cdot v-v$, where $g \in G$ and $v \in V$. By "abstract nonsense," $J_{G}$ is a right exact functor from $\mathcal{R}(G)$ to the category of $\mathbb{C}$-vector spaces. The following observation goes back to the works of Jacquet and Langlands.

Lemma 4.5 (see Prop. 2.35(b) in [3]). If $G$ is an $\ell_{c}$-group, the functor $J_{G}$ is exact. 
4.5. Representations of $\ell_{c}$-groups. Lemma 4.5 has important consequences for the representation theory of $\ell_{c}$-groups. Special cases of the following result have been used in the literature, but we include a proof for convenience.

Proposition 4.6. Let $G$ be an $\ell_{c}$-group. For any $\pi \in \mathcal{R}(G)$, the smooth dual $\pi^{\vee}$ is an injective object in the category $\mathcal{R}(G)$.

Proof. For any $\rho \in \mathcal{R}(G)$ we have a chain of natural isomorphisms

$$
\operatorname{Hom}_{\mathcal{R}(G)}\left(\rho, \pi^{\vee}\right) \cong \operatorname{Hom}_{\mathcal{R}(G)}\left(\rho \otimes_{\mathbb{C}} \pi, \mathbb{C}\right) \cong \operatorname{Hom}_{\mathbb{C}}\left(J_{G}\left(\rho \otimes_{\mathbb{C}} \pi\right), \mathbb{C}\right),
$$

and by Lemma 4.5, the right hand side is a composition of three exact functors in $\rho$.

Corollary 4.7. If $G$ is an $\ell_{c}$-group and $\pi \in \mathcal{R}(G)$ is admissible, then $\pi$ is injective in $\mathcal{R}(G)$.

Proof. Combine Lemma 4.3 with Proposition 4.6.

Corollary 4.8. If $G$ is an $\ell_{c}$-group such that every smooth irreducible representation of $G$ is admissible, then every nonzero smooth representation of $G$ has an irreducible quotient.

Proof. By Zorn's lemma, if $\rho \in \mathcal{R}(G)$ is finitely generated and $\rho \neq 0$, then $\rho$ has an irreducible quotient (no assumptions on $G$ are required here). Hence if $\rho \in \mathcal{R}(G)$ is arbitrary with $\rho \neq 0$, then $\rho$ has an irreducible subquotient. But in our situation, all smooth irreducible representations of $G$ are injective by Corollary 4.7, so we see that $\rho$ has an irreducible quotient.

4.6. The Pontryagin dual of an $\ell_{c}$-group. Let $U$ be an $\ell_{c}$-group. If $U$ is merely viewed as a topological group, one knows how to define its Pontryagin dual $U^{*}$; it is a commutative topological group which is canonically identified with the Pontryagin dual of the abelianization $U^{\mathrm{ab}}=U / \overline{(U, U)}$ (here $\overline{(U, U)}$ is the closure of the grouptheoretic commutator subgroup of $U)$.

However, for $\ell_{c}$-groups the Pontryagin dual construction has some special properties:

Lemma 4.9. If $U$ is an $\ell_{c}$-group, then every continuous homomorphism $\chi: U \longrightarrow \mathbb{C}^{\times}$ takes values in the unit circle $S^{1} \subset \mathbb{C}^{\times}$and has open kernel. The Pontryagin dual $U^{*}$, with its standard compact-open topology, is also an $\ell_{c}$-group.

Because of this lemma, in what follows, if $U$ is an $\ell_{c}$-group we will think of elements of the Pontryagin dual $U^{*}$ as homomorphisms $\chi: U \longrightarrow \mathbb{C}^{\times}$whose kernel is open in $U$, and we will refer to them as smooth homomorphisms.

Proof. If $\chi: U \longrightarrow \mathbb{C}^{\times}$is a continuous homomorphism, then $\chi(U)$ is equal to the union of its compact subgroups, whence $\chi(U) \subset S^{1}$. Moreover, $\chi$ has open kernel since a sufficiently small open neighborhood of $1 \in \mathbb{C}^{\times}$contains no subgroups of $\mathbb{C}^{\times}$ other than $\{1\}$. For the last statement we may assume, after replacing $U$ with $U^{\text {ab }}$, that $U$ is commutative. Given any compact open subgroup $K \subset U$, let $K^{\perp}$ denote its annihilator in $U^{*}$. Then $K^{\perp}$ is a compact open subgroup of $U^{*}$ (it can be identified with the Pontryagin dual of the discrete quotient $U / K)$. Subgroups of the form $K^{\perp}$ 
form a basis of neighborhoods of $1 \in U^{*}$ (here the assumption that $U$ is a filtered union of its compact open subgroups becomes crucial), and they also exhaust all of $U^{*}$ as $K$ gets smaller and smaller, by the previous remarks. This shows that $U^{*}$ is an $\ell_{c}$-group.

4.7. Summary of some results of F. Rodier. We conclude this section by stating some of the results appearing in [11] that are used in $\S 5$. Until the end of the section we fix a second countable $\ell$-group $G$ and a normal closed subgroup $U \subset G$ that is an $\ell_{c}$-group. The action of $G$ on $U$ by conjugation induces an action of $G$ on $U^{*}$ by topological group automorphisms.

If $(\rho, W)$ is a smooth representation of $U$ and $\chi: U \longrightarrow \mathbb{C}^{\times}$is a smooth homomorphism, then, following Rodier, we write $\widetilde{W}(\chi)$ or $\widetilde{\rho}(\chi)$ for $J_{U}\left(\rho \otimes_{\mathbb{C}} \chi^{-1}\right)$; more concretely, $\widetilde{\rho}(\chi)=\widetilde{W}(\chi)$ is the quotient of $W$ by the subspace spanned by all elements of the form $\rho(u) \cdot w-\chi(u) \cdot w$, where $u \in U$ and $w \in W$. If $S \subset U^{*}$ is a subset, we say that $(\rho, W)$ has spectral support contained in $S$ provided the kernel of the natural map

$$
W \longrightarrow \prod_{\chi \in S} \widetilde{W}(\chi)
$$

is injective. If this holds, then, in particular, $\rho(U)$ is commutative.

Remark 4.10. Rodier assumes in [11] that the subgroup $U$ itself is abelian. However, some of his theorems can be extended to the case where $U$ is not necessarily commutative simply by replacing $G$ with $G / \overline{(U, U)}$ and $U$ with $U / \overline{(U, U)}$. As this generalization is important for the applications we have in mind, we will use it to formulate the theorems below.

Note that if $(\pi, V)$ is a smooth representation of $G$ and $\chi \in U^{*}$, then $\widetilde{V}(\chi)$ inherits a natural action of the stabilizer $Z_{G}(\chi)$ of $\chi$ in $G$.

Theorem 4.11. Let $\chi \in U^{*}$ be such that the orbit $G \cdot \chi$ is locally closed in $U^{*}$. The functor $\pi \longmapsto \widetilde{\pi}(\chi)$ is an equivalence between the category of smooth representations of $G$ whose restriction to $U$ has spectral support contained in $G \cdot \chi$, and the category of smooth representations of $Z_{G}(\chi)$ on which $U$ acts via the scalar $\chi$. A quasi-inverse functor is given by $c-\operatorname{Ind}_{Z_{G}(\chi)}^{G}$.

This reduces at once to the case where $U$ is abelian, which follows from [11, Thm. 3, p. 186] and the remark on pp. 187-188 of op. cit.

Theorem 4.12. Let $(\pi, V) \in \mathcal{R}(G)$, and let $\chi \in U^{*}$ be such that its orbit $G \cdot \chi$ is closed in $U^{*}$ and $\left.\pi\right|_{U}$ has spectral support contained in $G \cdot \chi$. If $\widetilde{\pi}(\chi)$ is admissible as a representation of $Z_{G}(\chi)$, then $\pi$ is admissible as a representation of $G$.

This reduces at once to the case where $U$ is abelian, which is [11, Thm. 4, p. 189].

\section{Proof of Gutkin's conjecture}

In this section we complete the proofs of Theorems 1.1 and 1.3. The case where $F$ is either $\mathbb{R}$ or $\mathbb{C}$ was covered in $\S 2.2$, and the case where $F$ is finite was covered in $\S 2.3$. On the other hand, the statement of Theorem 1.3 formally makes sense in the case where $F$ is finite, provided we equip all the groups that appear in it with 
the discrete topology ${ }^{7}$. Since the only property of $F$ that plays a role in the proof presented below is the fact that $F$ is a locally compact totally disconnected self-dual topological field, we do not exclude the case where $F$ is finite for clarity.

5.1. Setup. We fix a (topological) field $F$ that is either finite and discrete, or local and nonarchimedean of arbitrary characteristic (with its standard topology). We will begin by proving Theorem 1.3 , and then indicate in $\S 5.5$ the changes needed to adapt our argument to Theorem 1.1. The proof given in $\S 5.5$ relies on the additional Remarks 5.2 and 5.4, which were included following the referee's suggestion.

Let $A$ be a finite dimensional associative nilpotent algebra over $F$, and let $\pi$ : $1+A \longrightarrow G L(V)$ be a smooth irreducible representation of $1+A$. If $\operatorname{dim}_{\mathbb{C}} V=1$, the statement of Theorem 1.3 becomes vacuous ${ }^{8}$, so we may assume that $\operatorname{dim}_{\mathbb{C}} V>1$. Our first goal is to realize $\pi$ via induction with compact supports from a subgroup of $1+A$ of the form $1+B$, where $B \subset A$ is an $F$-subalgebra of codimension 1 .

5.2. Key construction. Choose the smallest integer $m \geq 2$ such that the restriction of $\pi$ to $1+A^{m}$ acts on $V$ by scalars, and let $\zeta: 1+A^{m} \longrightarrow \mathbb{C}^{\times}$denote the corresponding smooth homomorphism. Note that $\zeta$ must be invariant under the conjugation action of $1+A$. In particular, Corollary 2.3 yields a pairing

$$
\bar{C}_{\zeta}:\left(1+\left(A / A^{2}\right)\right) \times\left(1+\left(A^{m-1} / A^{m}\right)\right) \longrightarrow \mathbb{C}^{\times}
$$

induced by the map

$$
(1+A) \times\left(1+A^{m-1}\right) \longrightarrow \mathbb{C}^{\times}, \quad(g, h) \longmapsto \zeta\left(g h g^{-1} h^{-1}\right) .
$$

Note that $\bar{C}_{\zeta}$ is not identically 1 , since otherwise $\pi\left(1+A^{m-1}\right)$ would consist of scalar operators, which would contradict the minimality requirement in the choice of $m$.

Note that the groups $1+\left(A / A^{2}\right)$ and $1+\left(A^{m-1} / A^{m}\right)$ can be canonically identified with the additive groups of the $F$-vector spaces $A / A^{2}$ and $A^{m-1} / A^{m}$. Therefore the Pontryagin dual of $1+\left(A^{m-1} / A^{m}\right) \cong A^{m-1} / A^{m}$ also has a canonical $F$-vector space structure: given $\lambda \in F$ and a smooth homomorphism $f: A^{m-1} / A^{m} \longrightarrow \mathbb{C}^{\times}$, we define $\lambda \cdot f: A^{m-1} / A^{m} \longrightarrow \mathbb{C}^{\times}$by $(\lambda \cdot f)(x)=f(\lambda \cdot x)$. Corollary 2.3(b) means that the pairing $\bar{C}_{\zeta}$ induces an $F$-linear map

$$
\Phi_{\zeta}:\left(A / A^{2}\right) \longrightarrow\left(A^{m-1} / A^{m}\right)^{*} .
$$

(The right hand side can be thought of either as the Pontryagin dual or as the $F$-vector space dual of $A^{m-1} / A^{m}$; the two can be identified because $F$ is self-dual.)

We see that the linear map $\Phi_{\zeta}$ is not identically zero, whence there exists a 1dimensional subspace $L \subset A^{m-1} / A^{m}$ such that the composition

$$
A / A^{2} \stackrel{\Phi_{\zeta}}{\longrightarrow}\left(A^{m-1} / A^{m}\right)^{*} \longrightarrow L^{*}
$$

is surjective, where the second map is given by restriction.

\footnotetext{
${ }^{7}$ In this setting the statements of Theorems 1.1 and 1.3 become essentially equivalent, in particular because for a finite discrete group every representation is smooth, and every irreducible representation is finite dimensional (hence admissible) and unitarizable by a standard averaging argument.

${ }^{8}$ The unitarizability of $\pi$ is equivalent to the statement that a smooth homomorphism $1+A \longrightarrow$ $\mathbb{C}^{\times}$takes values inside the unit circle in $\mathbb{C}^{\times}$, which follows from the fact that $1+A$ is an $\ell_{c}$-group (cf. Lemma 4.9).
} 
We let $A_{1} \subset A$ be the preimage of the kernel of the composition (5.1), and we let $U \subset A^{m-1}$ be the preimage of $L$. Note that $A_{1}$ and $U$ are two-sided ideals (hence also $F$-subalgebras) of $A$.

Lemma 5.1. We have $U \subset A_{1}$; equivalently, $\zeta$ annihilates the commutator subgroup $(1+U, 1+U)$ of $1+U$.

Proof. The assertion is vacuous if $m \geq 3$, so assume that $m=2$. We need to check that the commutator pairing $\bar{C}_{\zeta}:\left(1+\left(A / A^{2}\right)\right) \times\left(1+\left(A / A^{2}\right)\right) \longrightarrow \mathbb{C}^{\times}$is identically 1 on $1+L$. Using the fact that $\operatorname{dim}_{F} L=1$, we see that there exists $a \in U$ such that every element of $L$ is the image of $\lambda \cdot a$ for some $\lambda \in F$. But if $\lambda_{1}, \lambda_{2} \in F$, then $\lambda_{1} \cdot a$ and $\lambda_{2} \cdot a$ commute in $A$, whence $\bar{C}_{\zeta}\left(1+\lambda_{1} a, 1+\lambda_{2} a\right)=1$. This yields the lemma.

Remark 5.2. Suppose that, instead of the assumptions of $\S 5.1$, we have $F=\mathbb{R}$ or $F=\mathbb{C}$. Consider any continuous homomorphism $\zeta: 1+A^{m} \longrightarrow S^{1} \subset \mathbb{C}^{\times}$, where $m \geq 2$ and $\zeta$ not trivial on the commutator $\left(1+A, 1+A^{m-1}\right) \subset 1+A^{m}$. The construction of $\Phi_{\zeta}$ and $L, A_{1}, U$ can then be repeated verbatim, and the assertion of Lemma 5.1 remains valid, with the same proof.

5.3. Key auxiliary result. The next observation is key for applying the theorems of [11] in our situation.

Lemma 5.3. Let us keep all the notation of $§ 5.2$.

(a) There exists a smooth homomorphism $\chi: 1+U \longrightarrow \mathbb{C}^{\times}$such that $\left.\chi\right|_{1+A^{m}}=\zeta$.

(b) Any two such homomorphisms are $(1+A)$-conjugate.

(c) The stabilizer of any such $\chi$ in $1+A$ is equal to $1+A_{1}$.

Proof. (a) By Lemma 5.1, the restriction of $\pi$ to $1+U$ has a 1-dimensional subquotient. By Proposition 4.6, every smooth 1-dimensional representation of $1+U$ is injective as an object of $\mathcal{R}(1+U)$. Hence $\left.\pi\right|_{1+U}$ has a 1-dimensional quotient; say it is given by a smooth homomorphism $\chi: 1+U \longrightarrow \mathbb{C}^{\times}$. Since $\pi$ acts on $1+A^{m}$ via the scalar $\zeta$, we must have $\left.\chi\right|_{1+A^{m}}=\zeta$.

(b) Suppose $\chi_{1}, \chi_{2}: 1+U \longrightarrow \mathbb{C}^{\times}$are smooth homomorphisms with $\left.\chi_{1}\right|_{1+A^{m}}=$ $\zeta=\left.\chi_{2}\right|_{1+A^{m}}$. Then $\chi_{1}^{-1} \cdot \chi_{2}$ factors through $(1+U) /\left(1+A^{m}\right) \cong 1+L$. By our choice of $L$, there exists $x \in A$ with $\left.\Phi_{\zeta}(\bar{x})\right|_{L}=\chi_{1}^{-1} \cdot \chi_{2}$, where $\bar{x}$ is the image of $x$ in $A / A^{2}$. This means that

$$
\chi_{1}(1+y)^{-1} \cdot \chi_{2}(1+y)=\zeta\left((1+x)(1+y)(1+x)^{-1}(1+y)^{-1}\right)
$$

for all $y \in U$. Multiplying both sides by $\chi_{1}(1+y)$ and recalling that $\zeta=\left.\chi_{1}\right|_{1+A^{m}}$, we find that

$$
\chi_{2}(1+y)=\chi_{1}\left((1+x)(1+y)(1+x)^{-1}\right)
$$

for all $y \in U$, proving (b).

(c) A calculation similar to the one we just used shows that if $\chi: 1+U \longrightarrow \mathbb{C}^{\times}$ is a smooth homomorphism satisfying $\left.\chi\right|_{1+A^{m}}=\zeta$, then an element $1+x \in 1+A$ stabilizes $\chi$ if and only if $\zeta\left((1+x)(1+y)(1+x)^{-1}(1+y)^{-1}\right)=1$ for all $y \in U$. This is equivalent to $x \in A_{1}$ by the construction of $A_{1}$. 
Remark 5.4. Suppose again that, instead of the assumptions of $\S 5.1$, we have $F=\mathbb{R}$ or $F=\mathbb{C}$. Let $\zeta$ be as in Remark 5.2. Assertions (b) and (c) of Lemma 5.3 are also valid in this situation, with the same proofs. More precisely, the stabilizer in $1+A$ of any continuous homomorphism $1+U \longrightarrow S^{1}$ that extends $\zeta$ is equal to $1+A_{1}$, and any two such homomorphisms are $(1+A)$-conjugate.

5.4. Proof of Theorem 1.3. We remain in the setup of $\S 5.2$. By induction on $\operatorname{dim}_{F} A$, we may assume that all the assertions of Theorem 1.3 hold for any smooth irreducible representation of $1+A_{1}$. In particular, by Corollary 4.8 , the restriction $\left.\pi\right|_{1+A_{1}}$ has an irreducible quotient, say $\rho$. By construction, $\rho(1+U)$ commutes with $\rho\left(1+A_{1}\right)$, and hence, by Schur's lemma, the restriction of $\rho$ to $1+U$ is scalar, say, given by a smooth homomorphism $\chi: 1+U \longrightarrow \mathbb{C}^{\times}$.

Write $G=1+A$ and note that by Lemma 5.3(b), the orbit $G \cdot \chi$ is closed in the Pontryagin dual $(1+U)^{*}$, while by Lemma 5.3(c), the stabilizer $Z_{G}(\chi)$ equals $1+A_{1}$.

Since $\rho$ is irreducible, it is admissible by the induction hypothesis. Hence so is the smooth dual $\rho^{\vee}$ (cf. Lemma 4.3). Note that $1+U$ acts on $\rho^{\vee}$ via the scalar $\chi^{-1}$ and $Z_{G}\left(\chi^{-1}\right)=Z_{G}(\chi)=1+A_{1}$. Hence by Theorems 4.11 and 4.12 , the representation $\mathrm{c}-\operatorname{Ind}_{1+A_{1}}^{1+A}\left(\rho^{\vee}\right)$ of $1+A$ is irreducible and admissible. By Lemma 4.3 and Remark 4.4 , we have

$$
\operatorname{Ind}_{1+A_{1}}^{1+A} \rho \cong \operatorname{Ind}_{1+A_{1}}^{1+A}\left(\left(\rho^{\vee}\right)^{\vee}\right) \cong\left(\mathrm{c}-\operatorname{Ind}_{1+A_{1}}^{1+A}\left(\rho^{\vee}\right)\right)^{\vee},
$$

and by Lemma 4.3, the latter representation is admissible and irreducible. Hence $\operatorname{Ind}_{1+A_{1}}^{1+A} \rho$ is admissible and irreducible; in particular, the natural map $\mathrm{c}-\operatorname{Ind}_{1+A_{1}}^{1+A} \rho \longrightarrow$ $\operatorname{Ind}_{1+A_{1}}^{1+A_{1}} \rho$ must be an isomorphism. On the other hand, the natural map $\pi \longrightarrow$ $\operatorname{Ind}_{1+A_{1}}^{1+A_{1}} \rho$ coming from Frobenius reciprocity must also be an isomorphism; in particular, $\pi$ is admissible.

Next, by the induction hypothesis, the representation $\rho$ admits a $\left(1+A_{1}\right)$-invariant positive definite Hermitian inner product. Using a translation-invariant measure on $(1+A) /\left(1+A_{1}\right)$ and imitating the construction recalled in $\S 4.3 .3$ above, we can equip $\pi \cong \mathrm{c}-\operatorname{Ind}_{1+A_{1}}^{1+A} \rho$ with a $(1+A)$-invariant positive definite Hermitian inner product.

Finally, using an obvious transitivity property of the functors Ind and c-Ind and the induction hypothesis, we conclude that all the assertions of Theorem 1.3 hold for the representation $\pi$ of $1+A$. This completes the proof of Theorem 1.3.

5.5. Proof of Theorem 1.1. Let us end by explaining how the argument presented above needs to be modified in order to yield the assertion of Theorem 1.1. We begin by following the constructions of $\S 5.2$ almost verbatim (replacing $V$ with $\mathcal{H}$ ); everything there remains valid in the case where $\pi: 1+A \longrightarrow U(\mathcal{H})$ is a unitary irreducible representation. In order to complete the induction step we must show that there exists a unitary representation $\rho$ of $1+A_{1}$ such that $\pi \cong \mathrm{u}-\operatorname{Ind}_{1+A_{1}}^{1+A} \rho$.

To this end, we will replace references to results of [11] with the more classical "Mackey machine;" we refer the reader to [5, Chapter 6] for a more modern exposition. 
The restriction of $\pi$ to $1+U$ is a unitary (not necessarily irreducible) representation of $1+U$, which factors through the abelianization $(1+U)^{\text {ab }}$ by Remark 5.2. Therefore $\pi$ can be decomposed as a direct integral of 1-dimensional unitary representations of $1+U$, and this decomposition is determined by an $\mathcal{H}$-projection-valued measure $P$ on $(1+U)^{*}$. It is easy to see that in the language of $[5, \S 6.4]$, the triple $\left(\pi,(1+U)^{*}, P\right)$ is a "system of imprimitivity" on $1+A$. Moreover, since $\pi$ acts on $1+A^{m}$ via the character $\zeta$, we see that $P$ must be supported on the subset $S \subset(1+U)^{*}$ consisting of all $\chi$ such that $\left.\chi\right|_{1+A^{m}}=\zeta$. By Remark 5.4, $S$ is a single $(1+A)$-orbit. Thus $(\pi, S, P)$ is a "transitive system of imprimitivity" (op. cit., §6.5).

Finally, Theorem 6.31 in op. cit. implies that if $\chi \in S$, then $\pi$ can be obtained by unitary induction from a unitary representation of $Z_{1+A}(\chi)=1+A_{1}$ (Remark 5.4).

Remark 5.5. The proof we presented here only relies on the fact that $F$ is self-dual and not on the fact that it is totally disconnected. Thus we do obtain a uniform proof of Theorem 1.1 that is valid for all self-dual locally compact topological fields at once.

5.6. Final remarks. Under the hypotheses of Theorem 4.12, Rodier proved in [11, Thm. 5, p. 190] that if $\rho$ is a unitary irreducible representation of $Z_{G}(\chi)$ on which $U$ acts via $\chi$, then

$$
\left[\mathrm{u}-\operatorname{Ind}_{Z_{G}(\chi)}^{G} \rho\right]^{\mathrm{sm}}=\mathrm{c}-\operatorname{Ind}_{Z_{G}(\chi)}^{G}\left(\rho^{\mathrm{sm}}\right) .
$$

(As before, the reduction to the case where $U$ is abelian, which is treated in loc. cit., is immediate.) Using this result and analyzing the arguments of $\S \S 5.4-5.5$ above, we see that they imply

Proposition 5.6. Let $F$ be a local nonarchimedean field, let $A$ be a finite dimensional associative nilpotent algebra over $F$, and let $\pi: 1+A \longrightarrow U(\mathcal{H})$ be a unitary irreducible representation. Then the smooth part $\pi^{\text {sm }}$ is irreducible as a representation of $1+A$ (in the algebraic sense), and $\pi$ (equivalently, $\pi^{s m}$ ) is admissible.

It is not known to us whether the same result holds for more general $\ell$-groups of the form $\mathbb{G}(F)$, where $F=\mathbb{F}_{q}((t))$ and $\mathbb{G}$ is a unipotent algebraic group over $F$.

If $F$ is an infinite countable field ( $\operatorname{such}$ as $\mathbb{Q}$ ), we can equip $F$ with the discrete topology, so that it becomes a locally compact second countable topological field. Even though such an $F$ is not self-dual, the statements of Theorems 1.1 and 1.3 make sense in this setting as well. Justin Conrad asked whether their conclusions still hold. The answer is unknown to us. (Note, however, that they do hold when $A$ is commutative, since Schur's lemma can be applied in this case.)

\section{Acknowledgements}

My research was supported by the NSF grant DMS-0701106 and the NSF Postdoctoral Research Fellowship DMS-0703679. I thank Eugene Gutkin for inciting my interest in his conjecture, and Akaki Tikaradze for many stimulating discussions. I am grateful to Vladimir Drinfeld, from whom I learned the general strategies for studying representations of nilpotent groups that are employed in the article. I was also inspired by recent work of Jeff Adler and Alan Roche [1] on discrete series representations of unipotent groups over local fields. 
I would like to thank Eugene Gutkin and the anonymous referee for catching misprints and inaccuracies, as well as suggesting several improvements to the exposition.

\section{References}

[1] J. D. Adler and A. Roche, Discrete series representations of unipotent p-adic groups, J. Lie Theory 15 (2005), no. 1, 261-267.

[2] C. A. M. André, Irreducible characters of finite algebra groups, in Matrices and group representations (Coimbra, 1998), Vol. 19 of Textos Mat. Sér. B, 65-80, Univ. Coimbra, Coimbra (1999).

[3] J. N. Bernštein and A. V. Zelevinskii, Representations of the group $G L(n, F)$, where $F$ is a local non-Archimedean field, Uspehi Mat. Nauk 31 (1976), no. 3(189), 5-70.

[4] M. Boyarchenko and V. G. Drinfeld, A motivated introduction to character sheaves and the orbit method for unipotent groups in positive characteristic. ArXiv: math.RT/0609769, version 1.

[5] G. B. Folland, A course in abstract harmonic analysis, Studies in Advanced Mathematics, CRC Press, Boca Raton, FL (1995), ISBN 0-8493-8490-7.

[6] E. A. Gutkin, Representations of algebraic unipotent groups over a selfdual field, Funkcional. Anal. i Priložen. 7 (1973), no. 4, 80.

[7] Z. Halasi, On the characters and commutators of finite algebra groups, J. Algebra 275 (2004), no. 2, 481-487.

[8] I. M. Isaacs, Characters of groups associated with finite algebras, J. Algebra 177 (1995), no. 3, 708-730.

[9] A. A. Kirillov, Unitary representations of nilpotent Lie groups, Uspehi Mat. Nauk 17 (1962), no. 4 (106), 57-110.

[10] C. C. Moore, Decomposition of unitary representations defined by discrete subgroups of nilpotent groups, Ann. of Math. (2) $\mathbf{8 2}$ (1965) 146-182.

[11] F. Rodier, Décomposition spectrale des représentations lisses, in Non-commutative harmonic analysis (Actes Colloq., Marseille-Luminy, 1976), 177-195. Lecture Notes in Math., Vol. 587, Springer, Berlin (1977).

[12] G. van Dijk, Smooth and admissible representations of p-adic unipotent groups, Compositio Math. 37 (1978), no. 1, 77-101.

Mathematics Department, University of Michigan, 530 Church Street, Ann Arbor, Mi 48109-1043, USA

E-mail address: mityab@umich.edu 